\title{
Intergenerational contacts and Covid-19 spread Omnipresent grannies or bowling together?
}

\author{
Marco Albertini ${ }^{*}$, Lucas Sage ${ }^{2}$, Stefani Scherer ${ }^{3}$ \\ 1. Department of Political and Social Sciences, University of Bologna \\ Strada Maggiore 45, 40125 Bologna, Italy. marco.albertini2@unibo.it*Corresponding author.
}

2. GEMASS, Sorbonne University, Rue Pouchet 59-61, 75017 Paris, France. lucas.sage@etu.sorbonne-universite.fr

3. Department of Sociology and Social Research, University of Trento, Via Verdi 26, 38122, Trento, Italy. stefani.scherer@unitn.it

Keywords: COVID-19; Intergenerational Relations, Intergenerational Ties, Elderly 


\begin{abstract}
Many scientists are currently contributing research on SARS-CoV2, with social scientists focusing on demographic and behavioral aspects when it comes to the diffusion of the virus. Recent publications include valid contributions about the importance of population's demographic composition to understand country-differences in fatalities, and some speculations about the origins of different pace and patterns of diffusion. Among them the idea that intergenerational contacts would contribute to explain the fast spread and high fatality among the elderly population in some countries. We argument that in order to contribute to the scientific knowledge speculation is not enough and acknowledge that in the absence of solid, comparable data it is difficult to bring these ideas to an empirical test. Further, we present a simulation experiment shedding serious doubts on the importance of intergenerational contacts to spread the virus on the elderly population but underlining, instead, the importance of the high connectedness within the elderly population. That southern Europeans are not bowling alone seems to be more relevant to explain high diffusion among elderly than their contact to their (grand-)children.
\end{abstract}




\section{$1 \quad$ Introduction}

At the time in which we are writing the spreading of SARS-CoV2 virus has reached significant levels in a large number of countries and WHO has declared it a pandemic. Many scientists, from different disciplines, scrambled to provide explanations for the observed pattern of diffusion of the virus and its cross-countries variation, i.e.: the different paces of the epidemic, the composition of the affected population, as well as the different levels of the case fatality rate. Although we cannot assess at this point to what extent country differences are artificially due to different testing strategy adopted in the various countries (see our discussion below), it seems reasonable to assume that other social and demographic factors play a role. Social scientists, in particular, focused on the demographic, social and behavioural aspects of the epidemic (Mossong et al. 2008).

After the Hubei province in China, Italy was one of the first countries in the world where the spreading of SARS-CoV2 and the number of deaths associated with COVID-19 reached significant levels (WHO 2020; Robin-Champigneul, 2020, ISS 2020). Most importantly, since the very beginning of the epidemic, Italian data seem to suggest that not only was the spreading of the disease faster than in other European countries, but also was that the case fatality rate (CFR), at least in the initial phases, much higher than in other countries. Moreover, percentages of elderly people among the infected population seemed particularly high (Backhaus 2020). The obvious question arises where country differences in general and more specifically the over-representation of the elderly in some countries came from (Bayer and Kuhn, Voxeu.org, 20 March 2020). Various publications, finding also resonance in the media, proposed mainly two non-mutually exclusive socio-demographic drivers: (1) the demographic composition of the population, in particular the prevalence of an elderly population, and (2) the structure of social interactions, precisely patterns of intergenerational contact (Balbo et al. 2020; Bayer and Kuhn 2020; Dowd et al. 2020a, 2020b; WHO-China joint mission report 2020; Wu et al. 2020; ISS 2020, RKI 17/2020).

The role of intergenerational contacts in the spreading of SARS-CoV2 - especially between grandchildren and grandparents due to grand-parenting activities - was also stressed by Dowd et al. (2020b). Similarly, Balbo and colleagues (2020) suggest that the "strong family ties" (supposedly) characterizing Southern European countries represent an additional risk factor vis-à-vis the spreading of the epidemic and the fatality rate of COVID19. High fatality rates in some Southern European countries are thus suggested to be not only due to age-composition-effects, but also due to the overrepresentation of elderly among the infected population.

In the following we discuss this explanation and argue that claiming that patterns of intergenerational relations in Southern Europe are a key causal factor to explain higher fatality rates lacks empirical evidence as well as theoretical consistence. Although the argument fits well with common sense about Southern European societies, we suggest that an alternative mechanism could play a greater role: the density of social contacts among the elderly. To support our claim, we present an agent-based model of virus propagation in which we vary the social network structure connecting two generational groups. Our simulation experiments show that high intergenerational connectedness alone is not sufficient to rapidly contaminate a large fraction of the elderly. Only when we introduce social connectedness among the elderly does the virus infect a large fraction of the elderly. In these scenarios, the number of intergenerational ties does not seem to matter much, that is, only a few intergenerational ties are sufficient to rapidly generate high contamination rates among the elderly, and consequent high CFR levels. We therefore underline the call for more rigorous empirical research and cautiousness in interpretation others made before us (Belloc et al., 2020; Squazzoni et al. 2020). 
2 The explanandum, or: the need to establish the phenomenon

After China and South Korea, Italy (i) has been forerunner in the spreading of SARS-CoV2 virus and (ii) has one the highest case fatality rate registered among the affected countries $(13.8 \%$ as of May $\left.5^{\text {th }} 2020^{1}\right)$. However, while the former fact is quite uncontested, the measurement of CFR and its comparison across countries is everything but unproblematic. Therefore, the existence of the phenomenon of markedly different CFR rates- to be explained by different patterns of intergenerational relations - is itself at stake.

\section{The limits to valid comparisons across contexts}

When it comes to documenting the spread of the epidemic COVID19 and CFR, cross-country comparisons are problematic. There are various accounts of the fact that the total number of deaths associated with COVID19 may be underestimated - in highly affected areas, numerous people died without being tested and these deaths do not show in the statistics. For instance, Italian data on excess mortality in the first quarter of 2020 suggest a surge of mortality for individuals beyond their 50ies (ISTAT 2020, RKI 2020). ${ }^{2}$ This bias is likely to be different across countries, regions and municipalities within the same countries, and at different periods during the evolution of the epidemic. Even more serious concerns about unbiased measurement and comparability of the data across contexts arise when looking at the denominator of CFR, making it a difficult task to arrive at meaningful conclusions. As a matter of fact, there are huge differences in the intensity of testing across countries and regions within the same country, and along the evolution of the epidemic. Two indicators such as the number of swabs per infected, or the number of swabs per 100 thousand population vary considerably along these dimensions. What is more, the strategy followed to select who has to be tested and who not is markedly different depending on the national or regional institutional context, and the point of the epidemic curve. A higher intensity of the testing activity is likely to be associated with an increase in the number of infected people - the denominator of the CFR - and thus lower CFR. Further, at similar levels of testing the selection strategy matters. For example, if also asymptomatic individuals are tested whose risk of dying of COVID19 is lower CFR declines. If swabs are taken predominantly from individuals with (severe) symptoms the denominator will only include individuals having high risk of dying of COVID19. And, given that the severity of COVID19 seems to increase with age, this per se leads to the elderly being over-represented in the statistics on the infected. As a consequence, parts of the phenomenon might simply be an artefact of the testing strategy and social scientists are still far from being able to firmly establish (one of the) the fact(s) to be explained (the explanandum), i.e. higher CFR of COVID19 in some countries.

What is more, at the time in which we are writing the exceptionalism of the Italian CFR data (vis-àvis the Chinese $5.5 \%$, South Korean $2.4 \%$ or the German case, $4.2 \%$ ) does not seem to be unique: CFR is high $(>11 \%)$ in the UK, Netherlands, Spain, France and Belgium. Moreover, important within-country differences exist. Data published by the ISS, ISTAT and Azienda Zero show that in fact in Italy individuals aged 50 or older are over-represented among the infected population, whereas those aged 70 or older are over-represented among the deceased population. However, the Veneto region adopted a much higher testing intensity since the very beginning of the epidemic, and (consequently) shows a much less pronounced age gradient of the infected population.

\footnotetext{
${ }^{1}$ For updated CFRs see also: https://coronavirus.jhu.edu/data/mortality

${ }^{2}$ https://towardsdatascience.com/covid-19-excess-mortality-figures-in-italy-d9640f411691
} 
3 The mechanisms, or: dissecting the phenomena

As mentioned, it was suggested that the explanation for extraordinarily high level of CFR observed in the early stages of the epidemic in Italy was to be found in the composition of the population and in the higher risk to be infected for elderly people. In the following we argue that even if we assume that there is in fact an explanandum, above-mentioned measurement issues aside, we still find it hard to firmly attribute such over-representation to patterns of intergenerational contacts.

As a matter of fact, not only are Italy (and Spain) not so different from other European countries when it comes to the likelihood of grand-parenting but actually have even lower likelihood of intergenerational social support exchange (Albertini 2016). Moreover, if intergenerational relations were a factor to explain different CFR then regions with very similar models of family relations such as Veneto and Lombardy - should show very similar patterns, but de facto register very different CFR (18.41 vs. $7.85 \%$ as of May $\left.7^{\text {th }}\right){ }^{3}$ In fact, as pointed out by Belloc and colleagues (2020), a within-country between-regions analysis would immediately refute the intergenerational contact hypothesis. An over-representation of elderly individuals among the infected population, is more compatible with an high intensity of age-homogeneous horizontal social relations - e.g. "bowling together" at the bocciofila or at the local bar than with age-heterogeneous networks in the context of a strong family tie system. ${ }^{4}$ The remaining part of the paper is devoted to providing theoretical foundation to this point.

\section{The model setup and network structure}

We simulate a SIR-type of model (Keeling and Rohani 2008) where in each period individuals are in one of the three status: Susceptible $(S)$ if they have not been infected yet; Infected $(I)$ if they carry the virus and can contaminate others; Recovered $(R)$ if they recovered from the virus, and can no longer spread it or receive it. The population size $\mathrm{N}$ remains constant along the simulation. We split the population into two demographic groups of equal sizes $(\mathrm{N} / 2)$ that represent generations. For simplicity, we call them young (G1) and elderly $(\mathrm{G} 2)$. To represent different family structures, we embedded agents in social networks of various topologies where nodes are individuals and ties represent a connection between individuals. We first create two separate random networks with normal degree distribution. One component connects young individuals among each other, and the other one the elderly. We label this type of ties within group tie, as opposed to intergenerational ties that connect individuals of different generations. We exogenously define the total number of ties $\mathrm{E}$ in the population. $\gamma E$ is the number within group ties of the elderly generation, and $(1-\gamma) E$ the number of within group ties of the young generation. Parameter $\gamma$ characterizes the sociability of the elderly, relatively to the one of the young. Intuitively, the higher the $\gamma$, the more "vivid" the social life of the elderly.

In a second step, $\delta$ ties are "rewired" meaning that $\delta$ ties replaced by intergenerational ties with $\delta \leq$ $N / 2$. This procedure, inspired by Watts and Strogatz' (1998) small world network model, builds bridges between the two generations, although in our model there is no within generation transitivity. When an intergenerational tie is built, the algorithm prioritizes individuals with no intergenerational ties, such that each-individual in the network, can have at maximum, one intergenerational tie, i.e. only one family.

We build different network structures to represent different scenarios, by manipulating $\delta$ and $\gamma$. To illustrate how, we depict two of the ideal-typical scenarios we are interested in. For $N=3000, \gamma=$ 0 and $\delta=1500$, the elderly have no contacts among themselves, but they are all connected to one young agent. This represents a world with strong intergenerational connections but a non-existent

\footnotetext{
${ }^{3}$ Official epidemic data from Italian Protezione Civile, retrieved from https://datastudio.google.com/u/0/reporting/91350339-2c97-49b5-92b8-965996530f00/page/RdlHB

4 Next, news reported bocciofile- or bar- related early outbreaks in Lombardy, Veneto, Emilia-Romagna (https://bologna.repubblica.it/cronaca/2020/03/25/news/coronavirus bologna altro focolaio in una bocciofila-

252251849/; https://www.corriere.it/cronache/20 febbraio_24/coronavirus-trovata-connessione-il-focolaio-codognoquello-vo-dace326a-56ea-11ea-b89d-a5ca249e9e1e.shtml)
} 
social life among the elderly. Instead when $\gamma=0.25$ and $\delta=200$, a significant fraction of total ties connect elderly among each other, but there are few family ties.

Model dynamic: spread of the virus

Initially all individuals are set to $S$ except one agent among the young generation that is set to $I$. By doing so we assume, similarly to Dowd et al. (2020), that the patient zero is a young, mobile adult. Therefore, the spread of the virus, can only begin from the young generation, and pass to the elderly. At each iteration, the infected individuals interact with their contacts (individuals to which they are connected). However, since an individual that is or that has already been contaminated (either $I$ or $R$ ) cannot be contaminated again, we restrict the realms of interactions to the $S_{\mathrm{s}}$. The virus gets transmitted ${ }^{5}$ with a probability $p . p$ is in fact the product of two different mechanisms: the first one is the probability to encounter with someone from the network; the second is the probability to pass the virus through the interaction. For simplicity, we collapse these two mechanisms together into one single parameter $p$ and assume that all individuals have the same risk of getting infected once exposed to an infectious contact. Differentiating these two mechanisms would be necessary if we wanted to assess, for example, the overall consequences of changes, such as wearing masks without decreasing the probability to interact. Since our purpose is purely illustrative, we believe that it is reasonable to stick to this simpler version, and hence to limit the number of parameters of the model. After $k$ iterations, an infected individual $I$ turns to $R$, meaning that she recovered from the virus, and can no longer contaminates others or be contaminated. The simulation stops once there are no more $I$ in the population, that is when all individuals are either $S$ or $R$.

\section{Assumptions}

We do not introduce any other form of structure in the social networks. Individuals have one family, interact at random within their groups, there is no transitivity, and the distribution of degree is symmetric. These assumptions are common to most models but violate our knowledge about the structure of real world social networks (Gilbert and Hamill 2009). We adopt these simplifying assumptions because our purpose is illustrative and specific to the arguments about intergenerational interaction outlined above, and not explanatory or predictive. Moreover, we are only interested in the diffusion processes, therefore we do not model the death of infected individuals. This assumption is reasonable because one of the peculiarities of the SARS-CoV-2 virus is that infected individuals can carry on interacting with other without manifest symptoms for a substantial amount of time.

\section{Experiments and parameter values}

We systematically vary our two parameters of interest $\gamma$ and $\delta$ as indicated in overview 1 . For each point in the parameter space, we create 4 replications of the network structure, and 15 replications of the contamination process starting from the same network but with different patient 0 . Overall, for each point in the parameter space, we get 60 data points. Our outcome of interest is the proportion of the elderly that got contaminated $\frac{R_{e}}{N / 2}$ at the end of each simulation run, and the number of iterations it took for the simulation to stop. We report the average of this value across the 60 similar simulation runs.

\footnotetext{
${ }^{5}$ Epidemiological models often use $\mathrm{R}_{0}$, the basic reproduction number, in fact a catch-all factor containing behavioural aspects, network structures as well as the biological characteristics of the pathogen. We disentangle here explicitly the aspects related to social interaction by varying the network structure.
} 
Overview 1: list of parameters and values

\begin{tabular}{|l|c|l|}
\hline Parameter & Symbol & Value \\
\hline Network structure: & & \\
\hline Population size & $N$ & 3000 \\
\hline Number of ties & $\mathrm{E}$ & 15000 \\
\hline Average degree & $2 \mathrm{E} / \mathrm{N}$ & 10 \\
\hline Contamination process: & & \\
\hline Probability to contaminate & $p$ & 0.02 \\
\hline Time remain contagious & $k$ & 20 \\
\hline Range variation parameters of interest: & & \\
\hline Proportion of ties rewired & $\delta$ & {$[1: 99,1] \cup[100: 500,10] \cup[600: 1500,100]^{6}$} \\
\hline Proportion of ties connecting elderly & $\gamma$ & {$[0: 0.5,0.05]$} \\
\hline
\end{tabular}

\section{$4 \quad$ Results}

Figure 1 plots the diffusion rate of the virus among the elderly across experimental conditions. Each axis represents one of the two mechanisms of interest. The x-axis corresponds to the number of intergenerational ties $\delta E$ (logarithmic scale). The y-axis reports sociability of the elderly generation, expressed as the proportion of total ties connecting elderly among each other. It goes from $\gamma=0$ where the elderly have no within group ties, to $\gamma=0.5$ where is it equivalent to the sociability of the young generation.

Figure 1 diffusion of the virus among the elderly across experimental conditions

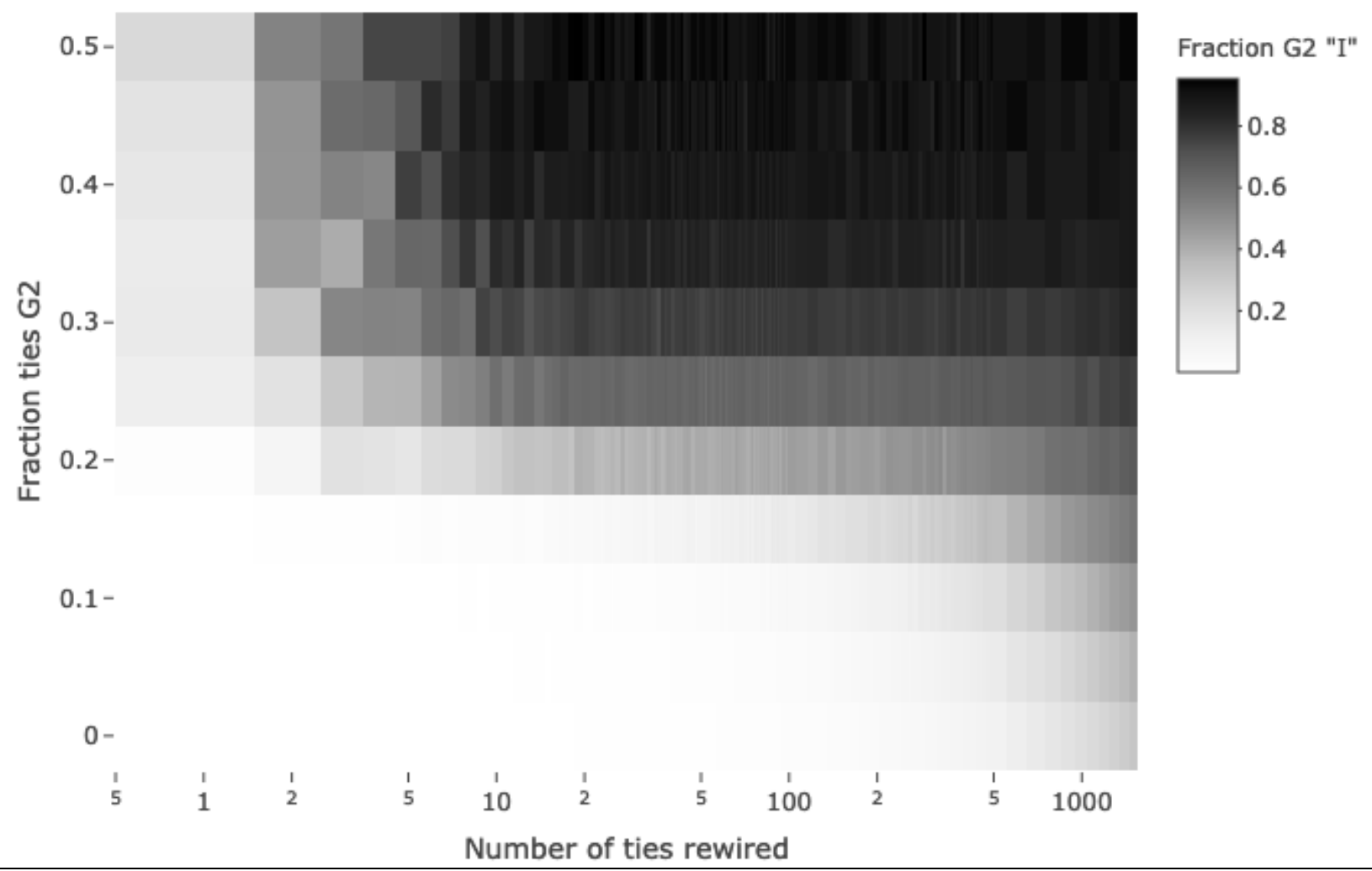

Note: the $\mathrm{x}$-axis (logarithmic scale) plots the number of intergenerational ties (number of rewired ties going from ingroup to out-group); the $\mathrm{y}$-axis $\gamma$ plots the fraction of total ties $\mathrm{E}$ of the elderly generation G2; the z-axis/color represents the proportion of the elderly that got contaminated at the end of the run averaged over 20 replications per condition.

\footnotetext{
${ }^{6}$ Notation of the interval work as follows: $[a: b, c]=a, a+c, a+2 c, \ldots, b-c, b$. In other words, $a$ is the lowest value, and $b$ the maximum value, while $\mathrm{c}$ is the value by which it increments.
} 
Our simulations reveal interesting findings. While it is clear that increasing the number of intergenerational ties, increases the share of infected elderly, these ties alone are not sufficient to largely propagate the virus among the elderly. Indeed, high contamination rates among this group largely depends on the proportion of within group ties $\gamma$. Even when the number of intergenerational ties is maximum, if $0.3 \leq \gamma \leq 0.5$ the diffusion rate among the elderly hardly exceeds 0.5 . On the contrary, we see that for high connectedness among the elderly, a few intergenerational ties are sufficient to contaminate a very large proportion of the elderly population. In fact, for $0.3 \leq \gamma \leq 0.5$, it seems like around 10 to 20 intergenerational ties are sufficient for the virus to largely infect the elderly. Put differently, there seems to be a threshold of intergenerational ties above which increasing the number of intergenerational ties does not lead to any significant increase in the proportion of elderly infected. Compared to the total number of ties $E$ in the network 15000, this threshold is remarkably small (around $0.67 \%$ to $1.33 \%$ of all ties). Although the value of this threshold depends on the value of $\gamma$, this threshold exists for all values of $\gamma \geq 0.3$. These results occur because, similarly to Watts and Strogatz (1998), only a few intergenerational ties are necessary to drastically reduce the average shortest path length. This effect is non-linear: while establishing a tie to the other group largely decreases the shortest path length when there are only a few of such ties, the effect becomes unnoticeable once a sufficient fraction of such ties already exists. Figure 2 illustrate this phenomenon. We plot the average shortest path length for a network with $\gamma=0.5$, in which we progressively rewire ties.

Figure 2: Average shortest path length as a function of the number of ties rewired

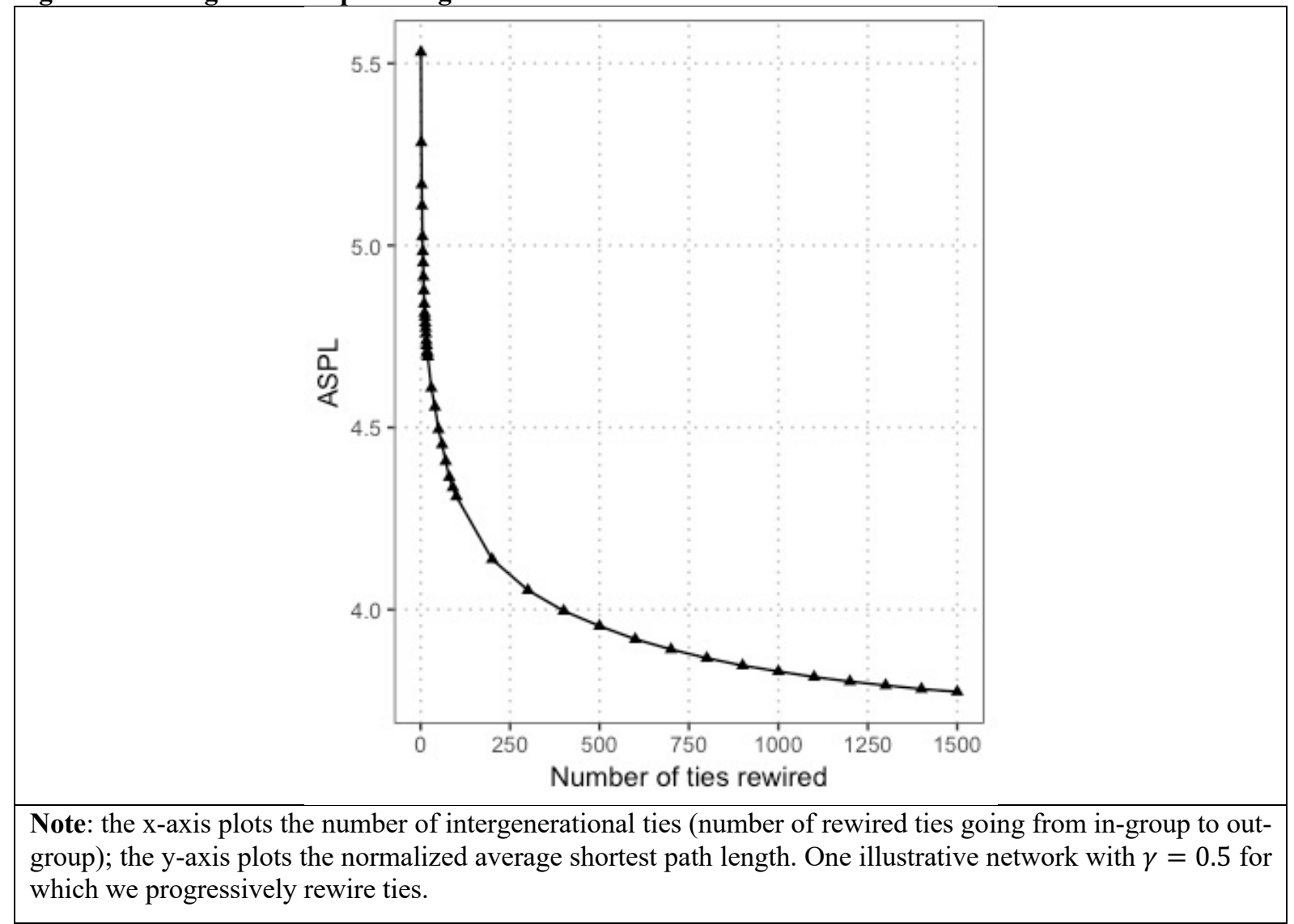

On the other hand, the sociability of the elderly $\gamma$ is a necessary condition to reach high proportions of the elderly infected, or, put differently, only a low in-group connectedness lowers substantively the risks of contamination among the elderly.

We can, therefore, tentatively conclude that, assuming the spread of the virus started from the young population, intergenerational ties are a necessary condition for the contamination of the elderly population, however, only a very small fraction of such ties are necessary. Above a threshold that is 
remarkably low, the amount of these ties is irrelevant. Differently, a significant level of horizontal ties in the elderly population (i.e. within-generation sociability) is a key necessary condition to explain a large spread of COVID19 within the elderly.

We now turn to how the network structure affects the speed of the propagation of the virus. Figure 3 plots this information, for the cases in which the virus infects a significant fraction of the elderly group. We select a threshold of $50 \%$, that is, we plot the conditions only when the virus contaminates at least half of the elderly.

\section{Figure 3: speed of diffusion across experimental conditions}

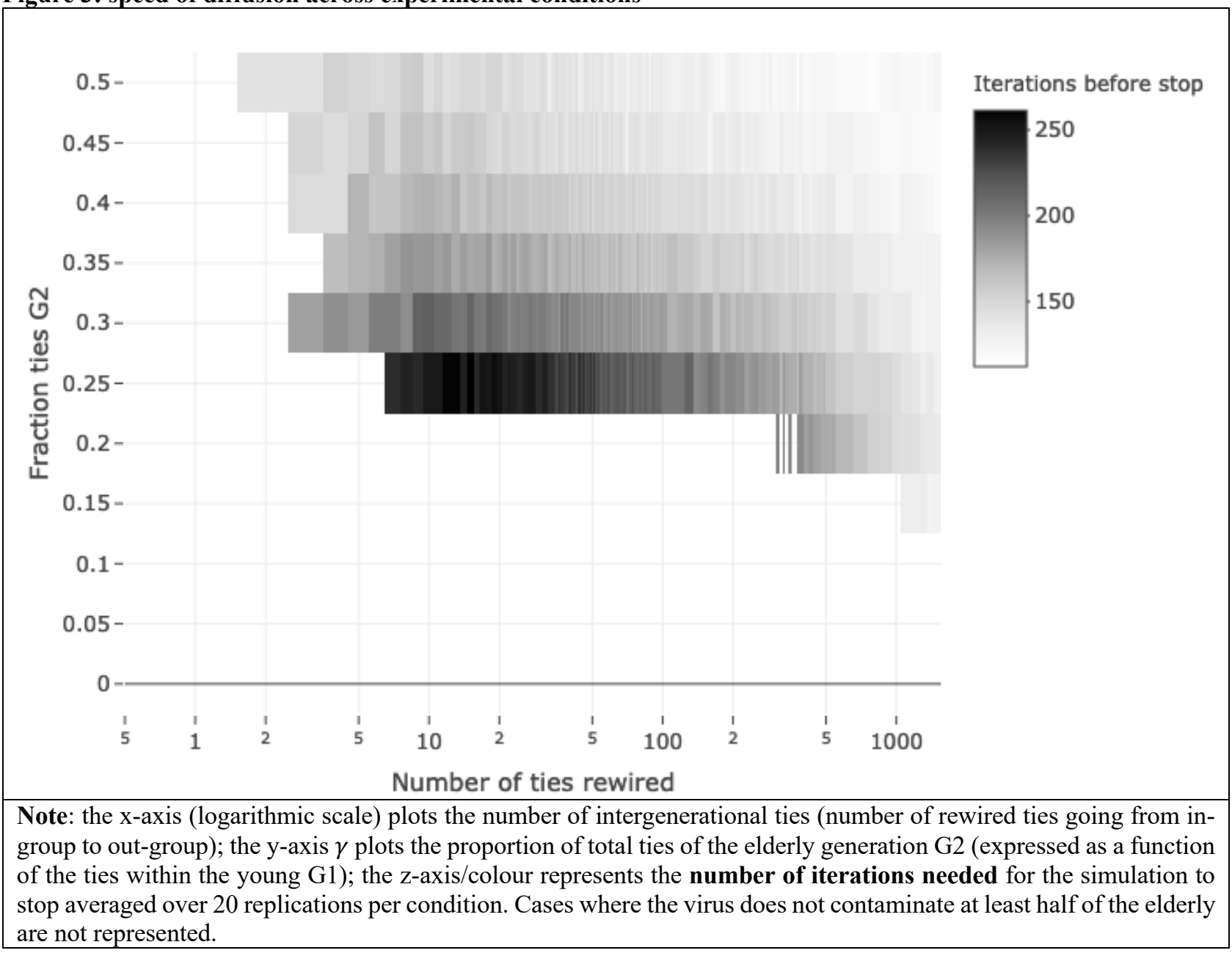

We see that the number of intergenerational ties accelerates the speed of the propagation, yet, the effect is weak. This is explained by the same mechanism as for the share of infected individuals among the elderly, that is, because only a few intergenerational ties are sufficient to drastically reduce the average shortest path length. 


\section{$5 \quad$ Conclusions: bocce not family!}

Recent publications provided some speculations about the reasons for documented country difference in pace and patterns of diffusion in COVID19. Some of them found some resonance in the wider public. Among them the idea that intergenerational contacts would contribute to explain the fast spread and high fatality among the elderly population in Italy (or southern Europe in general). In this contribution we point to the absence of solid, comparable data and the consequent difficulty to bring these ideas to a solid empirical test. Further, we presented a simulation experiment with the explicit purpose to test the mentioned claims, but do not pretend to arrive at detailed predictions. Simulations shed serious doubts on the importance of intergenerational contacts to spread the virus on the elderly population, but underline instead the importance of a high connectedness within the elderly population. That Southern Europeans are not bowling alone seems to be more relevant to explain high diffusion among elderly than their contact to their (grand-)children.

Our simulation experiments show that high intergenerational connectedness alone is not sufficient to rapidly contaminate a large fraction of the elderly. Only when we introduce social connectedness among the elderly does the virus infect large fractions of the elderly. In these scenarios, the number of intergenerational ties does not seem to matter much, that is, only a few intergenerational ties are sufficient to rapidly generate high contamination rates among the elderly.

We therefore underline the importance of the call for more rigorous empirical research and cautiousness in interpretations others made before us (Belloc et al., 2020; Squazzoni et al. 2020).

\section{References}

Albertini M., (2016). Ageing and family solidarity in Europe. Patterns and driving factors of intergenerational support. Policy Research Working Paper, 7678, Washington DC: World Bank.

Backhaus A. (2020) Coronavirus: Why it's so deadly in Italy. Medium, March 13. https://medium.com/@andreasbackhausab/coronavirus-why-its-so-deadly-in-italy-c4200a15a7bf

Balbo N., Billari F.C. and Melegaro A. (2020). The strength of family ties and covid-19. Context. https://contexts.org/blog/structural-shocks-and-extreme-exposures/\#balbo

Bayer C. and Kuhn M. (2020). Intergenerational ties and case fatality rates: A cross-country analysis. Vox CEPR Policy Portal, March 20, https://voxeu.org/article/intergenerational-ties-and-casefatality-rates

Belloc M., Buonanno P., Drago F., Galbiati R. and Pinotti P. (2020). Cross-country correlation analyss for research on COVID-19. Vox CEPR Policy Portal, March 28, https://voxeu.org/article/cross-country-correlation-analysis-research-covid19?fbclid=IwAR2AW4nRmTRgGoIw_DRZfEbDMtCeTKOQVT_gZaAqKqRGYOIe935jGppf RSA

Dowd J. B., Rotondi V., Andriano L., Brazel D. M., Block P., Ding X., Liu Y., Mills M. C. (2020a). Demographic science aids in understanding the spread and fatality rates of COVID-19, March 18, https://osf.io/m2ykh/?show=revision\&view only=c2f00dfe3677493faa421fc2ea38e295

Dowd J.B., Rotondi V., Andriano L., Brazel D. M., Block P., Ding X., Liu Y., Mills M. C. (2020b).

Demographic science aids in understanding the spread and fatality rates of COVID-19. PNAS,

117(18), 9696-9698. https://doi.org/10.1073/pnas.2004911117

Gilbert G. N. and Hamill L. (2009). Social circles: A simple structure for agent-based social network models. Journal of Artificial Societies and Social Simulation, 12(2), 1-3.

ISTAT (2020, May 4) Impact of the covid-19 epidemic on the total mortality of the resident population in the first quarter of $2020 \mathrm{https}: / / \mathrm{www}$.istat.it/it/files/2020/05/Istat-ISS -eng.pdf

Keeling M. J. and Rohani P. (2008). Modeling infectious diseases in humans and animals. Princeton University Press.

Mossong J. et al. (2008) Social Contacts and Mixing Patterns Relevant to the Spread of Infectious Diseases. PLOS Medicine, 5(3): e74 
RKI Robert Koch Institute (17/2020). Krankheitsschwere von COVID-19, Nowcasting: Erkrankungsfälle und Reproduktionszahl. Epidemiologisches Bulletin. https://www.rki.de/DE/Content/Infekt/EpidBull/Archiv/2020/Ausgaben/17_20.pdf? blob=publi cationFile

Robin-Champigneul F. (2020). COVID-19: comparaison du développement de l'épidémie et des mesures prises, entre 6 pays : Chine, Corée du Sud, Italie, France, Espagne et Allemagne. Laboratoir SAF, n. 17, April 5. https://www.ined.fr/fichier/rte/85/COVID19\%20en $\% 20$ France $\% 20-\% 20$ Comparaison $\% 20$ avec\%205\%20pays $\% 20-\% 20 \mathrm{~F} \% 20$ RobinChampigneul\%202020-04-05\%20v17.pdf

Squazzoni F., Polhill J. G., Edmonds B., Ahrweiler P., Antosz P., Scholz G., Chappin E., Borit M., Verhagen H., Giardini F. and Filbert N. (2020). "Computational models that matter during a global pandemic outbreak: A call to action”, Journal of Artificial Societies and Social Simulation, 23(2) 20. Doi: $10.18564 /$ jasss. 4298

Watts, D. J., \& Strogatz, S. H. (1998). Collective dynamics of 'small-world' networks. Nature, 393(6684), 440.

WHO (2020) Report of the WHO-China Joint Mission on Coronavirus Disease 2019 (COVID-19) https://www.who.int/docs/default-source/coronaviruse/who-china-joint-mission-on-covid-19final-report.pdf

Wu J.T., Leung K., Bushman M. et al. (2020) Estimating clinical severity of COVID-19 from the transmission dynamics in Wuhan, China. Nature Medicine, 26, 506-510. https://doi.org/10.1038/s41591-020-0822-7 